\section{Delirium in the oncology patient: A clinical dilemma}

\author{
By Tracey L. Das Gupta and Petrina N. MeGrath
}

\section{Abstract}

Delirium is a significant quality of life issue for which nurses are strategically positioned to prevent, identify, and provide intervention. Delirium occurs in $25 \%$ to $40 \%$ of patients with cancer, and may occur in as high as $85 \%$ of patients with advanced disease. However, a general lack of knowledge, an inability to distinguish varying manifestations and etiologies, and incomplete assessments have resulted in difficulty managing this phenomenon. This article reviews the literature on delirium, and examines the assessment and nursing interventions for care of the cancer patient. Recommendations for practice and research are made, emphasizing a holistic approach to enhance quality of life.

\section{Introduction}

The phenomenon of delirium is a significant quality of life issue for many persons with cancer, resulting in personal, social or financial consequences (Foreman, 1991). Delirium has been reported in $25 \%$ to $40 \%$ of cancer patients, with an increased incidence in advanced disease (Massie, Holland \& Glass, 1983). Patients with delirium have longer hospitalizations, require increased nursing supervision, and experience higher morbidity and mortality (Evans, Kenny \& Rizutto, 1993; Foreman, 1990; Fulop, Vita, Lyons \& Hammer, 1987).

Oncology nurses are in a strategic position to identify patients at risk for the development of delirium, detect early stages of delirium, and support patients and their families through this difficult and often life-threatening experience. Untreated delirium may progress to a permanent state of cognitive impairment further compromising quality of life. This paper will review the literature on delirium, and will examine the identification, assessment and interventions required for nursing care of cancer patients experiencing this phenomenon.

\section{The dilemma of defining delirium}

Defining delirium is a dilemma because of its fluctuating nature, widely varying and subtle clinical manifestations, and multiple etiologies. Currently, multiple terms (eg. acute confusion, acute brain failure, and dementia) which differ markedly are used inconsistently to describe delirium. These terms use

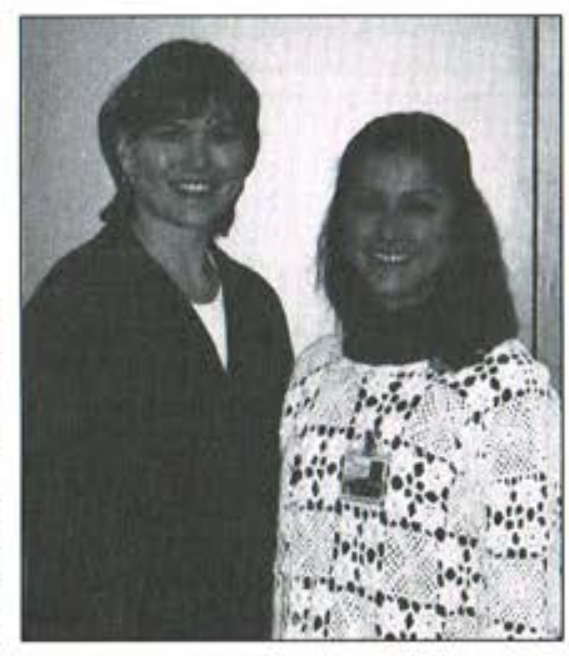

Petrina McGrath, left, and Tracey Das Gupta, right. various dimensions to define the same phenomenon.

Despite the variability in the conceptualization of delirium, there is general agreement regarding the features that comprise the phenomenon. These include concurrent disturbances in attention, perception, thinking, memory, psychomotor behaviour and sleep-wake cycle. The clinical manifestations differ from individual to individual, but typically develop along a continuum, are rapid, reversible and may fluctuate over the course of a day (American Psychiatric Association, 1994; Foreman, 1991; Lipowski, 1989; Zimberg \& Berenson, 1990).

For the purpose of this paper, delirium will be defined as a "pathophysiological disorder which manifests itself in impaired cognitive functioning and perceptual, emotional and behavioural alterations" (Zimberg \& Berenson, 1990, p.529). From a nursing perspective, this definition recognizes the physiological cause of delirium, while at the same time emphasizing that the manifestations may be both subjective and objective. As well, it reflects the holistic dimensions of the person, which is essential for the effective management of delirium and enhancement of quality of life.

\section{Prevalence of delirium}

Disparity in incidence and prevalence rates of delirium exist within the literature. These variations are the result of differences in the definition and measurement of delirium (Weinrich \& Sarnia, 1994), and lack of precise epidemiological studies (Muwaswes, 1993). Delirium has been estimated to occur in a range of $24 \%$ to $80 \%$ of hospitalized elderly (Cavanaugh, 1983; Foreman, 1990; Gillick, Serrell \& Gillick, 1982; Lipowski, 1983; Williams et al, 1985).

As cancer is largely a disease of the older person (Cohen, 1994) and given the often fragile state of the individual and the systemic effects of cancer and its treatment, the person with cancer is at an increased risk for the development of delirium (Zimberg \& Berenson, 1990). It has been noted to occur in as high as $85 \%$ of cancer patients with advanced disease (Massie, Holland \& Glass, 1983).

\section{Etiology of delirium}

The pathogenesis of delirium is poorly understood and is based on limited data. Hypotheses that account for the occurrence of delirium include: Reduction of cerebral oxidative metabolism, damaged neuronal enzyme systems, alterations in brain chemistry, neuronal loss,

\section{ABRÉGÉ}

\section{LE DÉLIRE CHEZ LE PATIENT EN ONCOLOGIE: UN DILEMME CLINIQUE}

Le délire est une question de qualité de vie pour laquelle les infirmières occupent une position stratégique en matière de prévention, d'identification et d'intervention. Le délire se produit chez 25 à $40 \%$ des patients vivant avec le cancer, un taux qui peut monter à $85 \%$ chez les patients atteints de cancers avancés. Toutefois, un manque général de connaissances, l'incapacité à distinguer les diverses manifestations et étiologies ainsi que des évaluations incomplètes ont rendu difficile la gestion de ce phénomène. Cette étude fait la recension de la littérature médicale sur le délire et examine l'évaluation et les interventions infirmières réalisées dans le cadre des soins au patient en oncologie. On y présente des recommandations relatives à la pratique et à la recherche tout en prônant une approche holistique de la santé en vue de rehausser la qualité de vie.

Tracey L. Das Gupta, RN, BScN, OCN, is currently educator/professional practice leader for medical/radiation/surgical oncology and Petrina N. McGrath, RN, BScN, OCN, is a professional practice leader for surgical oncology/general surgery, both at Sunnybrook Health Science Centre, North York, Ontario. 
decreased synthesis and function of neurotransmitters, and stress reaction (Adams, 1988; Foreman, 1986; Lipowski, 1983; Weinrich \& Sarnia, 1994; Zimberg \& Berenson, 1990). The signs and symptoms manifested by delirium are dependent on the etiological event (Muwaswes, 1993). Lipowski (1983) states that in $80 \%-95 \%$ of reported cases of clinically diagnosed delirium, organic etiological factors can be identified. Table One lists the common etiologies of delirium in the oncology population.

According to Foreman (1986), psychological and environmental factors are equally as important as physiological factors in the development of delirium. Table Two lists common psychological and environmental factors facing persons with cancer. Organic causes, for example, anemia, may increase a person's vulnerability to psychosocial stressors such as fatigue. These psychosocial stressors may facilitate the onset of delirium, and increase its severity or duration (Lipowski, 1983). Research, however, has not yet established whether organic and psychosocial etiologies are interrelated.

Individual differences in susceptibility have been found to influence the response to etiological factors. Increased age, advanced disease, and previous cognitive impairment are among risk factors found to predispose patients to delirium (Schor et al, 1992). It is important to note, although age may increase a person's vulnerability, the acceptance of confusion as a normal correlate of the aging process is inappropriate (Weinrich \& Sarnia, 1994). Delirium needs to be considered an indicator of an acute medical condition rather than an inevitable event. Failure to identify, diagnose and treat the underlying

\section{Table One: Organic etiologies of delirium}

\section{Etiology}

Tumour effects

Cancer treatment side effects (chemotherapy, radiation, biological response modifiers, surgery)

Age-related changes

Chemotherapy/

immunotherapy

Medications

Comorbid diseases

Nutritional status

\section{Examples}

- Space occupying lesions

- CNS metastases

- Cerebral edema

- Tumour lysis

- Progressive infections

- Metabolic abnormalities (ectopic hormones, SIADH, hypercalcemia)

- Infection and fever

- Fluid and electrolyte imbalances

- Organ failure

- Medication toxicity

- Compromised nutritional status

- Alteration in bowel and bladder

- Altered pharmacodynamics and increased chemo-related side effects as a result of changes in intestinal absorption, distribution, metabolism and excretion

Carmustine, vincristine, vinblastine, l'asparaginase, ifosfamide, intrathecal procarbazine, high dose methotrexate and Ara-C, $5 \mathrm{FU}$, cytarabine, cyclosporin, interleukin II, interferon, monoclonal antibodies

Psychotropic drugs, cardiac drugs (digitalis), GI (H2 antagonists), narcotic analgesia, anti-inflammatory drugs, antiemetics

COPD, endocrine disorders, hepatic disease, renal impairment

Anorexia, cachexia, vitamin deficiencies (B 12, folate), hypoglycemia

(Fleishman \& Lesko, 1989; Inaba-Roland \& Maricle, 1992; Lipowski, 1983; Palmieri, 1991; Weinrich \& Sarnia, 1994; Zimberg \& Berenson, 1990)

Table Two: Psychosocial etiologies of delirium

$\begin{array}{ll}\text { Etiology } & \text { Example } \\ \text { Psychological } & \text { - Severe emotional stress } \\ \text { - Depressive response } \\ \text { - Poor quality of sleep } \\ \text { - Decreased contact with friends and family } \\ \text { - Pre-existing conditions such as alcohol and drug abuse } \\ \text { - Changes in hearing and vision } \\ \text { - Bereavement } \\ \text { - Anticipatory grief } \\ \text { - Acute and chronic pain } \\ \text { - Situational disturbances } \\ \text { - Fatigue } \\ \text { - Unfamiliar environment creating a lack of meaning in the environment } \\ \text { - Sensory overload/deprivation } \\ \text { - Immobilization } \\ \text { Environmental Sleep deprivation } \\ \text { - Lack of temperospatial reference points }\end{array}$

(Foreman, 1986; Lipowski, 1983; Weinrich \& Sarnia, 1994) 
cause of delirium could result in serious or even lethal consequences (Lipowski, 1987).

There is a group of patients for which organic etiologies cannot be identified. Lipowski (1983) reports that this group represents 5\% to $20 \%$ of persons with delirium and refers to them as an "unsolved puzzle". Persons who would fall in this group include those who, as they near death, begin to speak in symbolic language that can be misinterpreted as delirium. They may speak of taking a trip, preparing to "go home", or may sometimes speak of finding themselves going back and forth between the present reality and another place or another world (Vachon, M.L.S., personal communication, October 18, 1995).

\section{Assessing delirium}

The assessment of delirium in the clinical setting requires a knowledge of risk factors and familiarity with etiological factors and their cognitive and behavioural manifestations. In addition, fine-tuned assessment skills and the use of bedside screening and measurement tools are essential to detect subtle changes indicative of delirium.

Palmateer and McCartney (1985) found that up to $72 \%$ of hospitalized elderly patients who experience delirium were never identified. Currently, a limited knowledge base about delirium, incomplete mental status assessments, and inappropriate use of terminology have resulted in the lack of identification and intervention for patients experiencing delirium (Inouye et al, 1990; Inaba-Roland \& Maricle, 1992; Rasin, 1990). When symptoms of delirium are identified, they are often misdiagnosed (Lipowski, 1983). Common features of delirium such as agitation, disorganized thinking, and disturbances in the sleep-wake cycle may mimic other psychiatric illnesses such as acute psychotic reaction, mania, dementia, schizophrenia or depression (Inaba-Roland \& Maricle, 1992). This may lead to inappropriate treatment and interventions.

Nurses most often use the term "confusion" to describe cognitive deficits (Palmateer \& McCartney, 1985). Their assessments are based almost exclusively on the patient's level of orientation (eg. patient oriented to person, place and time) and psychomotor behaviour (eg. patient pulling at tubes). When doing this, other manifestations of delirium are overlooked resulting in incomplete assessments (Inaba-Roland \& Maricle, 1992; Morency, 1990; Weinrich \& Sarnia, 1994).

Documentation of cognitive impaiment in clinical records often lacks behavioural descriptions and provides little of the detailed information needed to establish a diagnosis or treatment approach. The use of more specific terminology is required to differentiate delirium from major conditions such as dementia.

Differentiation between delirium and dementia is critical for an accurate diagnosis and effective treatment (Weinrich \& Sarnia, 1994). Both conditions are responsible for changes in mental status in the elderly, and each have their own specific symptoms, etiologies and expected outcomes (Morency, 1990). Level of consciousness and clinical course may be used to distinguish delirium from dementia. Alertness, which is usually unchanged in patients with dementia, may be reduced or increased in patients with delirium. Typically the symptoms of dementia are relatively stable, whereas symptoms of delirium develop over a short period of time and fluctuate (American Psychiatric Association, 1994).

Early identification of delirium is dependent upon the recognition of cognitive and behavioural manifestations. Disturbances in the sleep-wake cycle are often the first sign of its onset. Patients often appear drowsy and nap throughout the day, while experiencing periods of wakefulness during the night. Thinking is disorganized and fragmented, manifested by rambling and incoherent speech. There may be a reduction in a patient's ability to reason, judge, use abstract concepts, or problem-solve. The ability to sustain attention to a single stimuli is reduced and has an impact on short-term memory. Patients are often easily distracted, resulting in the impairment of registration, retention and retrieval of information. Perceptual disturbances such as hallucinations or illusions are common. Hallucinations may be visual, tactile or auditory (Rasin, 1990, Tueth \& Cheong, 1993).

The level of consciousness may be reduced or clouded in a patient with delirium, and can fluctuate between periods of lethargy and excitability. Psychomotor behaviour may alternate between periods of hyperactivity and hypoactivity. The above behaviours may fluctuate throughout the day and exacerbate in late afternoon or evening (Foreman, 1991; Morency, Levkoff \& Dick, 1994; Rasin, 1990; Tueth \& Cheong, 1993).

Although the literature provides detailed descriptions of the cognitive and behavioural manifestations of delirium, little data exist to support these as fundamental dimensions. Orientation and psychomotor behaviour, two aspects frequently utilized by nurses to detect delirium, have been found to be nonspecific in their ability to discriminate between persons with and without delirium. However, attention and concentration, features infrequently assessed, have been found to have a greater specificity. The discrepancy between what is believed to be useful in the assessment of delirium and what is actually of value may explain why many patients with delirium are never identified (Foreman, 1990, 1991).

\section{Instruments for assessment}

Increasingly, standardized screening instruments are being used to identify the presence and severity of delirium. Instruments may be classified as mental status questionnaires, psychomotor tests, or symptom rating scales. In selecting an appropriate instrument for clinical use, it is important that the instrument is valid, reliable, easy to use at the bedside, and places little demand on an acutely ill person.

Commonly used mental status questionnaires include the Mini Mental Status Exam (MMSE) (Folstein, Folstein \& McHugh, 1975), Short Portable Mental State Questionnaire (SPMSQ) (Pfeiffer, 1975), and the Cognitive Capacity Screening Exam (CCSE) (Jacob, Bernhard, Delgardo \& Strain, 1977). These questionnaires are best used as screening tools for generalized cognitive impairment to identify those patients who are in need of further assessment. Mental status questionnaires may be used on admission to provide baseline data, and

Table Three: Cognitive and behavioural manifestations of delirium

\section{Cognitive manifestations}

- Disorganized and fragmented thinking

- Reduction in ability to reason, judge, use abstract concepts or problem-solve

- Rambling and incoherent speech

- Reduced ability to sustain attention

- Decreased short-term and/or long-term memory

- Perceptual disturbances (hallucinations, illusions)

- Decreased or clouded level of consciousness

- Disorientation to person, place and/or time

\section{Behavioural manifestations}

- Disturbances in sleep-wake cycle

- Fluctuating periods of lethargy and excitability

- Psychomotor behaviour may alternate between hyperactivity and hypoactivity

(Foreman, 1991; Morency, Levkoff \& Dick, 1994; Rasin, 1990; Tueth \& Cheong, 1993) 
applied at regular intervals for patients thought to be at risk for the development of delirium.

Symptom rating scales such as the Delirium Rating Scale (DRS) (Trzepacz, Baker \& Greenhouse, 1988) or the Neecham Confusion Scale (Neelon, Funk, Carlson \& Champagne, 1989), can be used at regular intervals to identify delirium and monitor its severity and the patient's response to treatment.

Currently, the Mini Mental Status Exam provides the best method to evaluate behaviour over time, however this instrument is unable to differentiate delirium from other cognitive impairments. The Delirium Rating Scale, which is able to differentiate delirium from dementia and other cognitive impairments, requires further research to establish validity and reliability in the oncology population.

\section{Intervention}

The management of delirium begins with determining the etiologic cause. While the etiologic cause is sought, nursing interventions must focus on maximizing the comfort and safety of the patient (Fleishman \& Lesko, 1989). The interventions must be individualized for the patient and family, and are dependent upon the presenting manifestations of delirium.

A patient experiencing delirium may be a danger to himself, and a source of distress for families and staff (Lipowski, 1987; Fainsinger, Tapper \& Bruera, 1993). Often pressure for quick results from distressed family and staff leads to incomplete assessments and inappropriate management. The use of restraints and sedation for behavioural manifestations, although appropriate in some circumstances, may be used as a solution and prevent further assessment.

Few studies examining appropriate management for persons experiencing delirium exist, although the literature describes activities that have been helpful. Interventions focus on manipulating psychological and environmental variables considered to be etiologies of delirium. Manipulation of environmental factors includes such actions as the appropriate use of lighting to ensure a sensory environment that is neither overstimulating nor sensory deprived. In addition, the use of calendars, clocks, small familiar objects and seasonal decorations may help to decrease disorientation by providing personal meaning to the environment.

Psychosocial factors include ensuring that patients' seeing and hearing needs are met, encouraging frequent contact with supportive family and consistent caregivers, and encouraging patients to walk, read and participate in personal care. In addition, providing comfort measures that decrease pain, agitation or anxiety are important (Batt, 1989; Fleishman \& Lesko, 1989; Inaba-Roland \& Maricle, 1992; Massie et al, 1983; Morency, 1990; Rasin, 1990; Weinrich \& Sarnia, 1994; Zimberg \& Berenson, 1990).

There has been very little research to investigate nursing interventions for the prevention of delirium. Previous studies on prevention in medical/surgical patients have focused on interventions that promote reality orientation (Chatham, 1978; Williams et al, 1985). However, there is a need to examine interventions that prevent delirium by focusing on features other than orientation.

\section{Conclusion}

The phenomenon of delirium continues to be problematic for patients with cancer and their families. Delirium alters cognitive, behavioural and emotional aspects of the person. Family members who witness these changes often become anxious and frightened, impacting interpersonal relationships. There is a need to improve the prevention, early identification, assessment and intervention of this quality of life issue.

The first step to improve nursing care begins with education. By providing nurses with the necessary knowledge and assessment skills to detect delirium, they can better recognize the distinct cognitive and behavioural changes. "For nursing, the value of using bedside

Table Four: Instruments for assessment of delirium

Description of instrument

\section{Mental Status Questionnaires}

Brief cognitive screening instruments that use an interview format (MMSE, SPMSQ, CCSE)

\section{Psychomotor Tests}

Tests that attempt to measure attention, a core feature of delirium (hand-held tachistoscope, Trail-Making Test)

\section{Symptom Rating Scales}

Standardized rating scales that identify and measure symptoms of delirium (Delirium Rating Scale, NEECHAM)

\section{Strengths}

- Easy to administer at the bedside

- 5-15 minutes to perform

- Identify generalized cognitive impairment

- Valuable for obtaining baseline data

- Useful in research on delirium

- Attention is a core feature of delirium

- Make use of already collected data

- Quantitate severity of symptoms and can be used to monitor response to treatment

- Minimal patient burden

- Assess cognitive and behavioural manifestations

Delirium Rating Scale:

- Differentiates delirium from other

forms of cognitive impairment

\section{Limitations}

- Do not assess all dimensions of delirium

- Require verbal responses

- Do not differentiate between delirium and dementia

- Race, socioeconomic status and education have been found to have effects on MMSE test scores

- Do not measure all dimensions of delirium - Individuals with physical or sensory impairment may not be capable of completing the test

Delirium Rating Scale:

- Scoring relies on clinical judgment based on unstructured assessments

- Difficult to standardize

NEECHAM

- Not designed to detect delirium as defined by the criteria of the DSM-IV (1994)

(Folstein, Folstein \& McHugh, 1975; Jacob, Bernhard, Delgardo \& Strain, 1977; Levkoffet al, 1991; Neelon, Funk, Carlson \& Champagne, 1989; Pfeiffer, 1975; Trzepacz, Baker \& Greenhouse, 1988) 
instruments is not in the computation of a score so the patient can be categorized as cognitively impaired or non-impaired, it is in the ability to identify the specific deficits in cognitive functioning. With this information, individualized nursing interventions can be developed" (Rasin, 1990, p.913). However, further research is required to determine the most effective interventions.

Qualitative research is needed to examine the patients' and families' experiences of delirium. It is important that the person is the focus of care. Nurses must reflect on how they interact with the patient experiencing delirium. They must recognize the patient's perspective and listen to the emotional message (Anderson, Knutsson, Hallberg \&
Norberg, 1993). Through increased knowledge of delirium and understanding of the patient and family experience, it is hoped that nurses will be able to develop new approaches to manage delirium and enhance quality of life.

\section{Acknowledgement}

The authors would like to thank Mary Vachon, RN, PhD, and Margaret Fitch, RN, PhD, for their support and encouragement in the preparation of this manuscript.

\section{References}

Adams, F. (1988). Neuropsychiatric evaluation and treatment of delirium in cancer patients. Advanced Psychosomatic Medicine, 18, 26-36.

American Psychiatric Association. (1994). Diagnostic and Statistical Manual of Mental Disorders. (4th ed.). Washington, DC: Author.

Anderson, E.M., Knotsson, I.K., Hallberg, I.R. \& Norberg, A. (1993). The experience of being confused: A case study. Geriatric Nursing, 14(5), 242-247.

Batt, L.J. (1989). Managing delirium: Implications for geropsychiatric nurses. Journal of Psychosocial Nursing, 27(5), 22-25.

Cohen, H.J. (1994). Biology of aging as related to cancer. Cancer Supplement, 74(7), 2093-2100.

Cavanaugh, S. (1983). The prevalence of emotional and cognitive dysfunction in a general medical population using MMSE, GHQ and BDI. General Hospital Psychiatry, 5, 15-24.

Chatham, M.A. (1978). The effects of family involvement on patients' manifestations of postcardiotomy psychosis. Heart and Lung, 7, 995-999.

Evans, C.A., Kenny, P.J., Rizzuto, C. (1993). Caring for the confused geriatric surgical patient: Associated with higher mortality and a leading cause of nursing home placement, postoperative confusion must be addressed. Geriatric Nursing, 14, 237-241.

Fainsinger, R., Tapper, M. \& Bruera, E. (1993). A perspective on the management of delirium in terminally ill patients on a palliative care unit. Journal of Palliative Care, 9(3), 4-8.

Fleishman, S. \& Lesko, L.M. (1989). Delirium and dementia. In J.C. Holland \& J.H. Roland, Handbook of Psychooncology (pp 342-355). Oxford: University Press.

Folstein, M.F., Folstein, S.E. \& McHugh, P.R. (1975). Mini Mental State Examination. Journal of Psychiatric Research, 12, 189-198.

Foreman, M.D. (1986). Acute confusional states in hospitalized elderly: A research dilemma. Nursing Research, 35(1), 34-38.

Foreman, M.D. (1990). Complexities of acute confusion. Geriatric Nursing, 6(6), 136-139.

Fulop, G., Vita, J., Lyons, J.S. \& Hammer, J.S. (1987). Impact of psychiatric comorbidity on length of hospital stay for medical/surgical patients: A preliminary report. American Journal of Psychiatry, 144(7), 878-882.

Gillick, M.R., Serrel, N.A. \& Gillick, L.S. (1982). Adverse consequences of hospitalization in the elderly. Social Science and Medicine, 16, 1033-1038.

Inaba-Roland, K.E. \& Maricle, R.A. (1992). Assessing delirium in the acute care setting. Heart and Lung, 21(1), 48-55.

Inouye, S.K., VanDyck, C.H., Alessi, C.A., Balkin, S., Siegal, A.P. \& Horwitz, R.I. (1990). Clarifying confusion: The confusion assessment method a new method for detection of delirium. Annals of Internal Medicine, 113(12), 941-948.

Jacob, J.W., Bernhard, M.R., Delgardo, A. \& Strain, J.J. (1977). Screening for organic mental syndromes in the mentally ill. Annals of Internal Medicine, 86, 40-46.

Levkoff, S., Liptzin, B., Cleary, P., Reilly, C.H. \& Evans, D. (1991). Review of research instruments and techniques used to detect delirium. International Psychogeriatrics, 3(2), 253-271.
Lipowski, Z.J. (1983). Transient cognitive disorders (delirium, acute confusional states) in the elderly. American Journal of Psychiatry, 140(11), 1428-1434.

Lipowski, Z.J. (1987). Delirium (acute confusional states). Journal of American Medical Association, 258, 1789-1792.

Lipowski, Z.J. (1989). Delirium in the elderly patient. New England Journal of Medicine, 329(9), 578-582.

Massie, M.J., Holland, J., Glass, E. (1983). Delirium in terminally ill cancer patients. American Journal of Psychiatry, 140(8), 1048-1049.

Morency, C.R. (1990). Mental status change in the elderly: Recognizing and treating delirium. Journal of Professional Nursing, 6(6), 356-365.

Morency, C.R., Levkoff, S.E., Dick, K.L. (1994). Research considerations: Delirium in hospitalized elders. Journal of Gerontological Nursing, 8, 24-30.

Muwaswes, M. (1983). Alterations in Consciousness. In V. Carrieria-Kohlman, A.M. Lindsey \& C.M. West (Eds.) Pathophysiological Phenomena in Nursing: Human Responses to Illness. Philadelphia: Saunders Company.

Neelon, V.J., Funk, S.F., Carlson, J.T. \& Champagne, M.T. (1989). The Neecham confusion scale: Relationship indicators of acute confusion in hospitalized elders. The Gerontologist, 29, 65A.

Palmateer, L.M. \& McCartney. (1985). Do nurses know when patients have cognitive deficits? Journal of Gerontological Nursing, 11 6-16.

Palmieri, D.T. (1991). Clearing up the confusion: Adverse effects of medications in the elderly. Journal of Gerontological Nursing, 17(10), 32-35.

Pfeiffer, E. (1975). A short portable mental status questionnaire for the assessment of organic deficit in elderly patients. Journal of American Geriatric Society, 31 266-268.

Rasin, J.H. (1990). Confusion. Nursing Clinics of North America, 25(4), 909-919.

Schor, J.D., Levkoff, S.E., Lipsitz, L.A., Reilly, C.H., Cleary, P.D., Rowe, J.W. \& Evans, D.A. (1992). Risk factors for delirium in hospitalized elderly. JAMA, 267(6), 827-831.

Tueth, M.J., Cheong, J.A. (1993). Delirium: Diagnosis and treatment in the older patient. Geriatrics, 48(3), 75-80.

Trzepacz, P.T., Baker, R.W. \& Greenhouse, J. (1988). A symptom rating scale for delirium. Psychiatry Research, 23, 89-97.

Weinrich, S. \& Sarnia, L. (1994). Delirium in the older person with cancer. Cancer Supplement, 74(7), 2079-2091.

Williams, M.A., Campbell, E.B., Rayner, W.W., Musholt, M.A., Mlynarczyk, S.M. \& Crane, L.F. (1985). Predictors of acute confusional states in hospitalized elderly patients. Research in Nursing and Health, 8, 31-40.

Wolanin, M.O. (1981). Physiologic aspects of confusion. Journal of Gerontological Nursing, 7(4), 236-242.

Zimberg, M. \& Berenson, S. (1990). Delirium in patients with cancer: Nursing assessment and intervention. Oncology Nursing Forum, 17(4), 529-538. 